\title{
Monolithic high-contrast grating based polariton laser
}

\author{
Seonghoon Kim, ${ }^{\dagger}$ Zhaorong Wang, ${ }^{\dagger}$ Sebastian Brodbeck, ${ }^{\ddagger}$ Christian Schneider, ${ }^{\ddagger}$ \\ Sven Höfling,,$\$$ \\ $\dagger$ Department of Electrical Engineering and Computer Science, Ann Arbor, MI 48109, USA \\ $\ddagger$ Technische Physik, Am Hubland, Würzburg 97074, Germany \\ \SUPA, School of Physics and Astronomy, St Andrews KY16 9SS, United Kingdom \\ $\S$ Department of Physics, Ann Arbor, MI 48109, USA \\ E-mail: dengh@umich.edu
}

\begin{abstract}
High-reflectance gratings promise new generations of vertical-cavity lasers that allow flexible control of the lasing mode properties and better integration. To date, conventional and polariton vertical-cavity lasers have been created with high-contrast gratings surrounded by air. Suspended in air, these gratings are delicate to fabricate and use, and are difficult for precise property control, electrical injection or thermal management. Here, we demonstrate a high quality vertical-cavity laser with a nonsuspended monolithic grating. High reflectance is achieved to allow the strong coupling regime, verified by angle-resolved photoluminescence measurements. We demonstrate polarized polariton lasing, manifested by a super-linear increase in the polariton emission and linewidth narrowing. We also demonstrate multi-wavelength operation of the polariton laser, with the wavelength controlled by design.
\end{abstract}




\section{Keywords}

polariton lasers, VCSEL, high-contrast gratings, photonic crystals

Vertical-cavity surface-emitting lasers (VCSELs) incorporating high-contrast subwavelength gratings (HCGs) instead of distributed Bragg reflectors (DBRs) have been intensively studied in the past decade, ${ }^{1,2}$ for they allow a more compact structure, high polarization selectivity, and control over the reflection-phase ${ }^{3}$ and thus the cavity mode properties. ${ }^{4,5}$ Furthermore, HCGs define the lateral dimensions of the cavity, which help achieve singlemode lasing for both photon lasing ${ }^{6}$ and polariton lasing ${ }^{2}$ and also enable straightforward creation of coupled cavity systems. ${ }^{7}$ However, HCGs make use of high-index-contrast to achieve high reflectance; they are often surrounded by air and require relatively complex fabrication processes to remove the sacrificial layer under the HCG layer. ${ }^{1}$ Suspended, they are susceptible to vibrational instability, ${ }^{8}$ inefficient in heat dissipation, and difficult for electrical injection, although there are unique advantages such as electromechanical tuning of cavity resonance. ${ }^{5}$ Recently, it was shown that monolithic HCGs (MHCGs) that are not suspended in air may also allow high reflectance. ${ }^{9,10}$ Such MHCGs would allow the same design flexibilities as HCGs while circumventing the main difficulties of HCGs. They can be fabricated in a precisely controllable way, have no mechanical instability, and allow efficient

heat dissipation as well as electrical contacts directly on the grating. ${ }^{11}$ In this letter, we report a MHCG-cavity polariton laser. High MHCG reflectance and thus high cavity quality factor is achieved using a two-layer MHCG structure, enabling the strong coupling regime and polariton lasing. Furthermore, we show the ability to control resonance energy of the cavity, and thus lasing frequency, by grating parameters.

The working principle of MHCG can be explained by the two-mode canceling theory, same as the conventional HCG. ${ }^{12}$ In the near sub-wavelength regime, the field in the grating layer can be decomposed into two waveguide-array (WGA) modes, the 0th and 2nd order modes for normal incidence, as odd modes are forbidden by symmetry and higher-order 
(b)
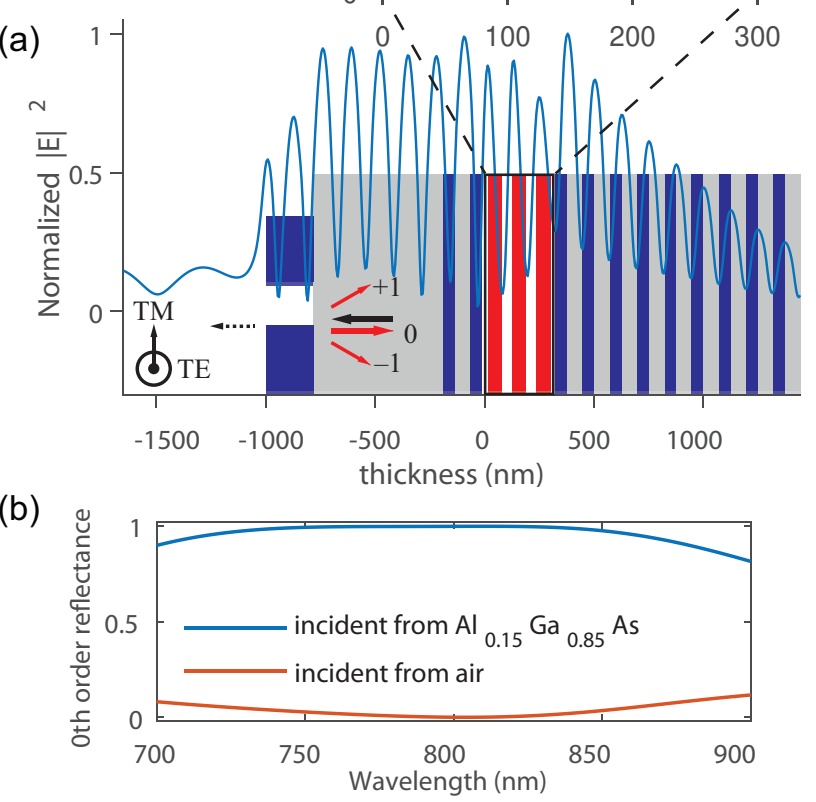

Figure 1: The MHCG cavity. (a) A schematic of the sample structure and the simulated TM-polarized electric field intensity profile. The sample consists of from left to right: a $\mathrm{Al}_{0.15} \mathrm{Ga}_{0.85} \mathrm{As}$ MHCG (blue), a $\mathrm{Al}_{0.85} \mathrm{Ga}_{0.15} \mathrm{As}$ spacer (grey), 1.5 pairs of top DBR made of $\mathrm{Al}_{0.15} \mathrm{Ga}_{0.85} \mathrm{As} / \mathrm{Al}_{0.85} \mathrm{Ga}_{0.15} \mathrm{As}$ (blue/grey), an AlAs cavity (white) with three stacks of GaAs QWs (red), and a bottom DBR. Light incident on the MHCG from inside the cavity (black arrow) will mostly be reflected (thick red arrow) with a small fraction diffracted to higherorder modes (thin red arrows), while transmission is negligible (black dotted arrow). (b) Simulated 0th order reflectance of MHCG, showing high asymmetry for light incident from the two sides of the MHCG. MHCG is highly reflective for the TM-polarized light incidence from the cavity side (blue) but shows low reflectance for light incident from air (red).

modes are cut off. We write $\mathcal{E}_{m}(x)$, the lateral profile of the WGA mode as:

$$
E(x, z)=\sum_{m=0,2} \mathcal{E}_{m}(x)\left[a_{m} e^{-j \beta_{m}\left(z-t_{g}\right)}+b_{m} e^{+j \beta_{m}\left(z-t_{g}\right)}\right]
$$

Here the exponential parts represent the forward and backward propagations in the $\mathrm{z}^{-}$ direction with coefficients of $a_{m}$ and $b_{m}$, respectively. $\beta_{m}$ is the propagation constant for $m$ th mode and $t_{g}$ is the thickness of grating. The two modes are mutually orthogonal in the grating layer, but can couple at the grating boundaries, $\boldsymbol{b}=\boldsymbol{R} \boldsymbol{a}$, where $\boldsymbol{R}$ is a $2 \times 2$ reflection matrix. It has large off-diagonal elements due to the abrupt change of refractive 

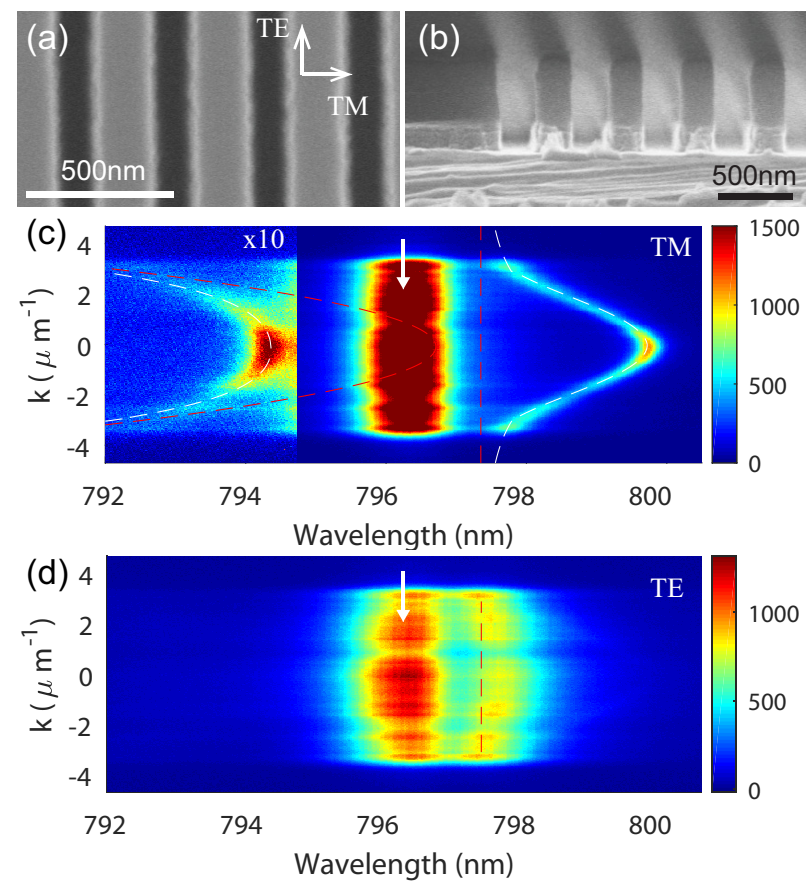

Figure 2: Experimental realization of MHCG microcavities. (a), (b) Scanning electron microscope (SEM) images of the top and side views of a fabricated MHCG, respectively. (c), (d) Momentum-space PL spectra of the TM-polarized and TE-polarized emission, respectively. Color indicates intensity. In (c), the spectral intensities at shorter wavelength than $794.5 \mathrm{~nm}$ in (c) is multiplied by 10 for better visibility of the UP dispersion. The red dashed lines indicate the exciton resonance measured from (d) and the cavity dispersion calculated based on the LP, UP and exciton energies. The white dahsed lines indicate the calculated LP and UP dispersions. The white arrows in (c), (d) mark the exciton resonance which is not strongly coupled to the cavity and therefore appear in both TE and TM (see main text for details).

index profile at the grating-air boundary. The coupling of the two modes helps equalize their amplitudes. The relative phase between the two modes can be controlled precisely by tuning the thickness of grating. Hence destructive interference between the two modes can be achieved at the output plane where the zeroth order diffraction mode is coupled out of the grating (black dotted arrow in Fig. 1(a)), leading to zero transmission and therefore $100 \%$ reflection.

Compared to conventional HCGs, MHCGs have a higher index material on one side, which may support non-evanescent higher-order diffraction modes (red arrows in Fig. 1(a)). In most applications, especially vertical cavities, only the zeroth order reflection is desirable. 
Therefore, the grating needs to be carefully engineered to minimize the energy carried away by the higher-order diffractions. We use the rigorous coupled wave analysis (RCWA) to find the parameters to ensure high reflectance of the MHCGs for transverse-magnetic (TM) polarization, with the electric field vector perpendicular to the grating grooves. The MHCG remains low reflectance for light of transverse-electric (TE) polarization. The simulated electric field intensity distribution in the cavity and the 0th order reflectance are shown in Fig. 1(a) and (b).

A peculiar feature of the MHCG is the high asymmetry of the reflectivity. Because of the existence of higher-order diffraction modes, the reflectance of the MHCG is high when the light is incident from the cavity side but low when the light is incident from the free-space side, as we illustrate in Fig. 1(b). This allows a high quality factor for the cavity with large field enhancement while at the same time efficient optical pumping from the free-space side.

High-reflectance MHCGs have been realized experimentally in recent studies, ${ }^{10,13}$ although the measured reflectance of the fabricated MHCG was lower than expected from the simulation. This is mainly because making ideal grating structures requires vertical side wall profiles, precise control of etching depth, and a flat etched surface. It is difficult to satisfy these conditions when the MHCG layer is on a substrate of the same material. ${ }^{9,10}$ Achieving strong coupling and efficient lasing requires a near-perfect MHCG with very high reflectance. We realize this by using a two-layer MHCG structure created by selective dry etching.

The MHCG-microcavity structure used in this work is shown in Fig. 1(a). The MHCG layer is made of $200 \mathrm{~nm}$ of $\mathrm{Al}_{0.15} \mathrm{Ga}_{0.85} \mathrm{As}$ on top of $600 \mathrm{~nm}$ of $\mathrm{Al}_{0.85} \mathrm{Ga}_{0.15} \mathrm{As}$ spacer, followed by 1.5 pairs of top DBR, a $\lambda / 2$ cavity with 12 quantum wells, and 36 pairs of bottom DBR. The two-layer MHCG structure allows selective etching of the $\mathrm{Al}_{0.15} \mathrm{Ga}_{0.85} \mathrm{As}$ grating layer which stops at the $\mathrm{Al}_{0.85} \mathrm{Ga}_{0.15}$ As layer underneath. The etching gas consists of $\mathrm{BCl} 3$ and SF6. A reaction between SF6 and AlGaAs forms $\mathrm{AlF}_{\mathrm{x}}$, which prevents etching. Therefore the etching rate is highly dependent on the composition of Aluminium. ${ }^{14}$ Since the first layer also has $15 \%$ of Aluminium, the selective etching process results in slanted vertical wall 
profiles. Therefore we etch the first layer using the nonselective etching process without SF6 and etch the residual first layer using the selective etching process. With this process, we create gratings with vertical side walls, precise etching depth determined by the thickness of the first layer, and a flat etched surface, as shown in Fig. 2(b). The periods of the fabricated gratings range from $328 \mathrm{~nm}$ to $344 \mathrm{~nm}$ and the duty cycles range from 0.6 to 0.7 .

Optical characterization of the sample was performed at 10K. The objective lens with a numerical aperture of 0.4 was used for both focusing the excitation laser on the sample and collecting the photoluminescence (PL). The sample was nonresonantly excited by a continuous wave laser at $790 \mathrm{~nm}$. The laser was chopped by an electro-optic modulator with $1 \%$ duty cycle at $10 \mathrm{kHz}$ to reduce sample heating. The PL is sent to the grating-based

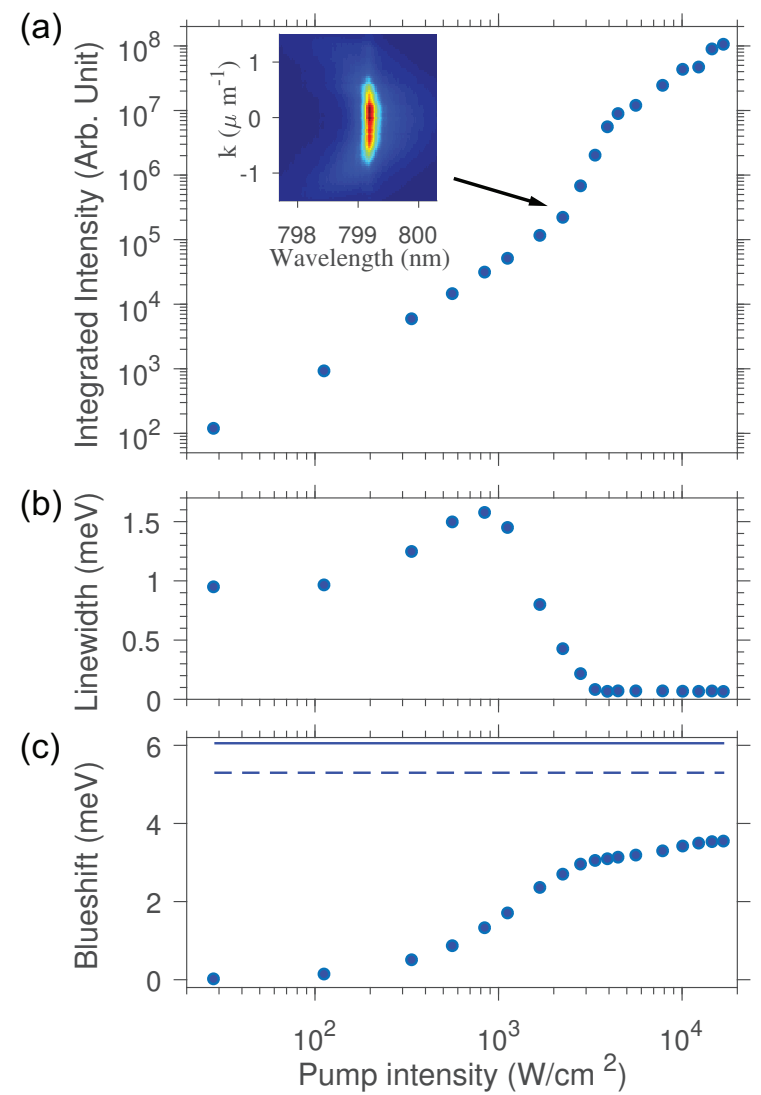

Figure 3: Characteristics of polariton lasing. (a) The integrated output intensity (b) linewidth and (c) blueshift of the lasing mode as a function of the pump intensity. The linewidth measurement is limited by the resolution of the monochromator of $0.03 \mathrm{~nm}$. The inset in (a) is the momentum space PL spectrum at the threshold pump intensity. The solid and dashed lines in (c) indicate the uncoupled cavity and exciton resonances, respectively. 
(b)
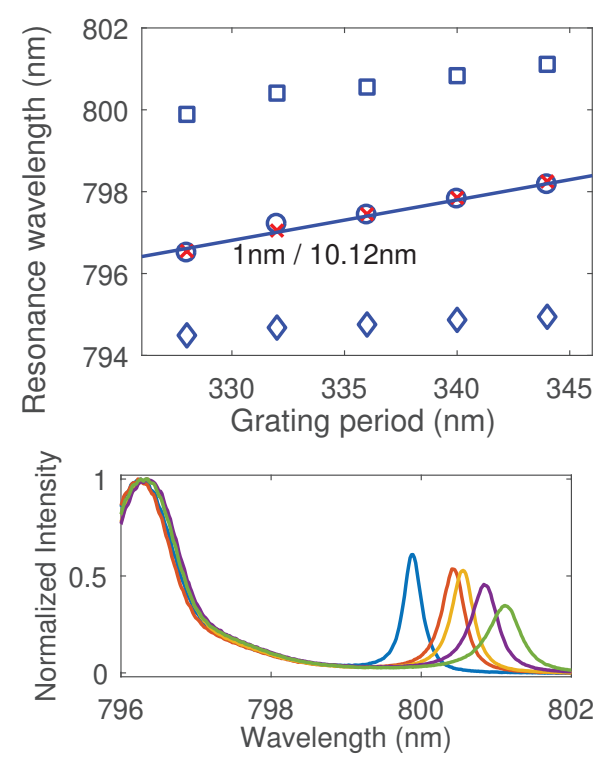

(c)

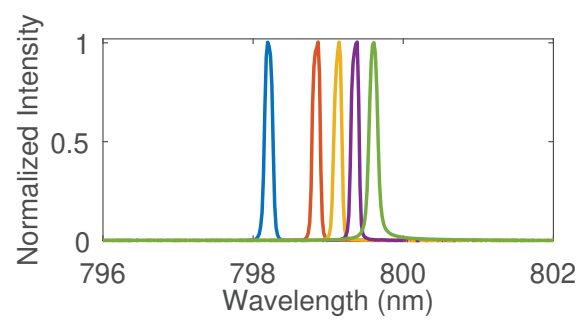

Figure 4: Tuning of the emission wavelength by the grating period. (a) The measured LP and UP resonances (open diamonds and square, respectively) at low pump powers and the corresponding cavity resonances as a function of the grating period. The cavity resonance obtained from the measured LP, UP, and exciton resonances are marked by the open circles, while the cavity resonance calculated by RCWA are marked by the red crosses. The solid line is a linear fit to the experimental cavity resonance vs period, showing tuning of the cavity resonance with the grating period at $1 \mathrm{~nm} / 10.12 \mathrm{~nm}$. (b) Emission spectrum at low pump powers as the grating period of the MHCG is varied from $328 \mathrm{~nm}$ to $344 \mathrm{~nm}$ with 4 nm increment. The LP resonance shifts toward longer wavelengths as the period increases. The exciton resonance remains the same in all the spectra. (c) The emission spectra of the lasing state at high pump powers for the corresponding MHCGs as in (b).

monochromator for spectroscopy.

Strong coupling is evident from the angle-resolved PL at low pump powers that shows dispersions of the lower polariton (LP) branch and upper polariton (UP) branch in TM polarization, centered in k-space at $\mathrm{k}=0$ at $799.7 \mathrm{~nm}$ and $794.3 \mathrm{~nm}$, respectively (Fig. 2(c), (d)). The TE-polarized PL shows emission from excitons at around $797.5 \mathrm{~nm}$, which are not strongly coupled to the cavity modes as the reflectance of MHCG is low for TE. The existence 
of two exciton lines could be caused by interlayer variations of $\mathrm{QWs},{ }^{15}$ as we confirmed by measurements of exciton emission from the unprocessed, planar part of the same wafer. The exciton mode around $796 \mathrm{~nm}$ shown in TM polarization is possibly from QWs that are not overlapped with electric field maxima inside the cavity and therefore are not coupled to the cavity. This is consistent with the simulated electric field distribution inside the cavity shown in Fig. 1(a), where the field maxima is slightly off-centered from QWs. From the measured LP, UP, and TE exciton energies at $\mathrm{k}=0$, we obtain the normal mode splitting of $10.2 \mathrm{meV}$ and exciton-photon detuning of $2.4 \mathrm{meV}$. Here, we use the exciton energy from the exciton emission appearing only at TE polarization. The calculated cavity resonance and exciton resonance from TE are indicated by red and white lines respectively in Fig. 2(c).

The power-dependent input-output relationship is shown in Fig. 3. We observe a nonlinear increase in the emission intensity at the threshold pump intensity of $2,000 \mathrm{~W} / \mathrm{cm}^{2}$, accompanied by the linewidth narrowing of the lasing mode at $k=0$. The spectrum at the threshold is shown in the inset of Fig. 3(a), where the polaritons mostly relaxed into $k=0$. The linewidth above the threshold approaches quickly to the resolution limit of about $0.03 \mathrm{~nm}$, as shown in Fig. 3(b). The lasing mode blueshifts smoothly as the pump power increases (see Fig. 3(c)) due to the polariton-polariton interactions, while remaining well below both the exciton and cavity energies. These results clearly show polariton lasing characteristics of the microcavity. The threshold intensity is comparable to other polariton devices. ${ }^{16}$ We observed similar lasing phenomena from MHCG devices with different cavity resonances which confirms the robustness of high quality MHCGs of varying parameters.

Finally, we demonstrate the tuning of the cavity resonances and polariton laser energy with respect to the grating period (Fig. 4). Unlike with DBRs, it is possible to engineer the reflection phase of the HCG by tuning grating parameters. The reflection phase of the mirror determines the phase matching condition for the cavity modes and therefore directly influences the cavity resonance. Here, we tune the reflection phase by changing the grating periods. As shown in Figure 4(a)-(b), the LP and UP resonances redshift as 
the grating period increases from $328 \mathrm{~nm}$ to $344 \mathrm{~nm}$. Using the measured LP, UP and TE exciton energies, we calculate the corresponding cavity resonance energy, which changes approximately linearly with the period. A linear fit yields $1 \mathrm{~nm}$ of resonance shift per 10.12 $\mathrm{nm}$ of change in the grating period. This result is in excellent agreement with the RCWA simulation indicated by the cross marks in Fig. 4(a). Here we fixed the duty cycle at 0.7 as measured by SEM.

Polariton lasing is achieved in all the gratings across the tuning range. The lasing wavelength for different periods are shown in Fig 4(c). We use the same pump intensities for different devices to make sure that the lasing wavelengths are not affected by the blueshift caused by the exciton reservoir. Above the threshold, the linewidths become narrower and emission from the different MHCGs become well separated spectrally. This would be important for applications such as on-chip multiwavelength laser arrays for wavelength division multiplexing. ${ }^{17}$

It is important to note that the tuning can also be achieved by changing the duty cycle. Therefore, a wide range of resonance tuning is possible by changing both the period and duty cycle of the grating, both of which can be controlled to nanometer accuracy with e-beam lithography. In this particular sample, it is possible to tune the cavity resonance from $784 \mathrm{~nm}$ to $808 \mathrm{~nm}$ while maintaining a quality factor above 1,000 based on RCWA simulations.

In conclusion, we show polariton lasing from vertical-cavity structures incorporating MHCGs. The PL measurement clearly shows a strong coupling regime and polariton lasing characteristics. The cavity resonance is controlled by grating parameters which can be systematically and accurately controlled by e-beam lithograhpy. The simple fabrication process of MHCGs is important for realizing a practical VCSEL based on HCGs with a possibility of making electrical contacts directly on HCGs due to its monolithic nature. ${ }^{11}$ Resonance tuning capabilities may be used for multiwavelength VCSEL arrays for wavelength division multiplexing. ${ }^{17}$ This may also be useful for HCG based polariton lattices and periodic chains ${ }^{18}$ which require accurate control of detuning between polariton sites. 


\section{Acknowledgement}

S.K., Z.W. and H.D. acknowledge the support by the National Science Foundation (NSF) under Awards DMR 1150593 and the Air Force Office of Scientific Research under Awards FA9550-15-1-0240. C.S., S.B. and S.H. acknowledge the support by State of Bavaria and the Deutsche Forschungsgemeinschaft (DFG) within the project SCHN11376 3-1. The fabrication of the SWG microcavities was performed in the Lurie Nanofabrication Facility (LNF) at Michigan, which is part of the NSF NNIN network.

\section{References}

(1) Huang, M. C. Y.; Zhou, Y.; Chang-Hasnain, C. J. A surface-emitting laser incorporating a high-index-contrast subwavelength grating. Nat. Photonics 2007, 1, 119-122.

(2) Zhang, B.; Wang, Z.; Brodbeck, S.; Schneider, C.; Kamp, M.; Höfling, S.; Deng, H. Zero-dimensional polariton laser in a subwavelength grating-based vertical microcavity. Light: Sci. Appl. 2014, 3, e135.

(3) Mateus, C. F. R.; Huang, M.; Deng, Y.; Neureuther, A. R.; Chang-Hasnain, C. Ultrabroadband mirror using low-index cladded subwavelength grating. IEEE Photonics Technol. Lett. 2004, 16, 518-520.

(4) Wang, Z.; Zhang, B.; Deng, H. Dispersion Engineering for Vertical Microcavities Using Subwavelength Gratings. Phys. Rev. Lett. 2015, 114, 073601.

(5) Huang, M. C. Y.; Zhou, Y.; Chang-Hasnain, C. J. A nanoelectromechanical tunable laser. Nat. Photonics 2008, 2, 180-184.

(6) Huang, M. C. Y.; Zhou, Y.; Chang-Hasnain, C. J. Single mode high-contrast subwavelength grating vertical cavity surface emitting lasers. Appl. Phys. Lett. 2008, 92, 171108. 
(7) Zhang, B.; Brodbeck, S.; Wang, Z.; Kamp, M.; Schneider, C.; Höfling, S.; Deng, H. Coupling polariton quantum boxes in sub-wavelength grating microcavities. Appl. Phys. Lett. 2015, 106, 051104.

(8) Yang, W.; Gerke, S. A.; Ng, K. W.; Rao, Y.; Chase, C.; Chang-Hasnain, C. J. Laser optomechanics. Sci. Rep. 2015, 5, 13700.

(9) Gebski, M.; Dems, M.; Szerling, A.; Motyka, M.; Marona, L.; Kruszka, R.; Urbańczyk, D.; Walczakowski, M.; Palka, N.; Wójcik-Jedlińska, A.; Wang, Q. J.; Zhang, D. H.; Bugajski, M.; Wasiak, M.; Czyszanowski, T. Monolithic high-index contrast grating: a material independent high-reflectance VCSEL mirror. Opt. Express 2015, 23, 11674-11686.

(10) Marciniak, M.; Gebski, M.; Dems, M.; Haglund, E.; Larsson, A.; Riaziat, M.; Lott, J. A.; Czyszanowski, T. Optimal parameters of monolithic high-contrast grating mirrors. Opt. Lett. 2016, 41, 3495-3498.

(11) Czyszanowski, T.; Gebski, M.; Dems, M.; Wasiak, M.; Sarzala, R.; Panajotov, K. Subwavelength grating as both emission mirror and electrical contact for VCSELs in any material system. Sci. Rep. 2017, 7, srep40348.

(12) Chang-Hasnain, C. J.; Yang, W. High-contrast gratings for integrated optoelectronics. Adv. Opt. Photonics 2012, 4, 379.

(13) Lee, J.; Ahn, S.; Chang, H.; Kim, J.; Park, Y.; Jeon, H. Polarization-dependent GaN surface grating reflector for short wavelength applications. Opt. Express 2009, 17, $22535-22542$.

(14) Lee, J. W.; Devre, M. W.; Reelfs, B. H.; Johnson, D.; Sasserath, J. N.; Clayton, F.; Hays, D.; Pearton, S. J. Advanced selective dry etching of GaAs/AlGaAs in high density inductively coupled plasmas. J. Vac. Sci. Technol., A 2000, 18, 1220-1224. 
(15) Damen, T. C.; Shah, J.; Oberli, D. Y.; Chemla, D. S.; Cunningham, J. E.; Kuo, J. M. Dynamics of exciton formation and relaxation in GaAs quantum wells. Phys. Rev. B 1990, 42, 7434-7438.

(16) Steger, M.; Fluegel, B.; Alberi, K.; Snoke, D. W.; Pfeiffer, L. N.; West, K.; Mascarenhas, A. Ultra-low threshold polariton condensation. Opt. Lett. 2017, 42, 1165-1168.

(17) Karagodsky, V.; Pesala, B.; Chase, C.; Hofmann, W.; Koyama, F.; ChangHasnain, C. J. Monolithically integrated multi-wavelength VCSEL arrays using highcontrast gratings. Opt. Express 2010, 18, 694-699.

(18) Nalitov, A.; Liew, T.; Kavokin, A.; Altshuler, B.; Rubo, Y. Spontaneous Polariton Currents in Periodic Lateral Chains. Phys. Rev. Lett. 2017, 119, 067406. 


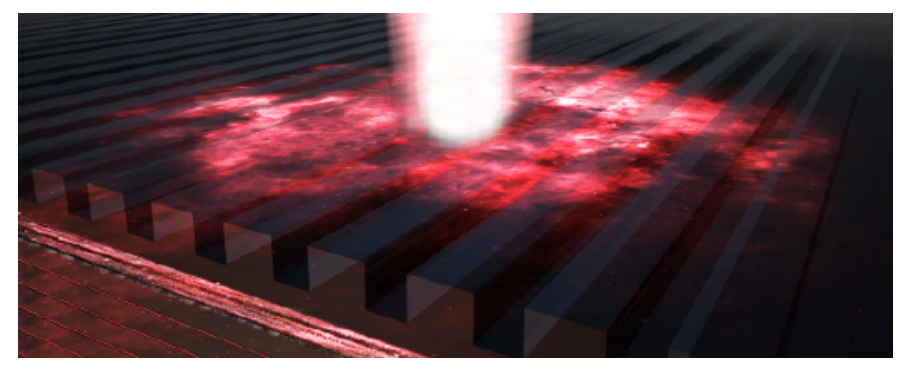

Figure 5: Table of content 